\title{
Cyclic lipodepsipeptides: time for a concerted action to unlock their application potential?
}

\author{
Niels Geudens ${ }^{1}$, Davy Sinnaeve ${ }^{1}$ \& José C Martins*,1 \\ ${ }^{1}$ Department of Organic \& Macromolecular Chemistry, Ghent University; Campus Sterre, S4, Krijgslaan 281, B-9000 Gent, Belgium \\ * Author for correspondence: jose.martins@ugent.be
}

\author{
"Apparently, the CLP blueprint can produce multifaceted biological agents, and much \\ fundamental insight remains to be gained in the correlation between CLP sequence, structure and \\ biological activity."
}

First draft submitted: 13 December 2017; Accepted for publication: 13 December 2017; Published online: 7 February 2018

\begin{abstract}
Keywords: antimicrobial peptides • cyclic lipopeptides • membrane interaction • natural product chemistry •
\end{abstract} oligomerization • structure-activity relationship

The range of antimicrobial peptides (AMPs) is very broad and covers a large and diverse group of molecules produced across different kingdoms of life. In a sense, all peptides that possess any activity against any microorganism can be classified as an AMP. Typically however, the AMP label is associated with oligopeptide chains of moderate length featuring two or more positively charged residues. Therefore, the AMP label is often considered synonymous to 'cationic antimicrobial peptides', although anionic AMPs also exist, such as maximin $\mathrm{H} 5$ or dermcidin. AMPs can be linear or cyclic, the latter typically involving disulfide bonds or the polycyclic thioether amino acid lantionine [1,2]. Quite characteristically, most AMPs only adopt a specific structure or more rigid conformation upon interaction with a membrane, while being flexible and unstructured in solution.

Cyclic lipopeptides (CLPs) can be considered as a subdivision of the AMPs, produced by nonribosomal peptide synthetases in various bacterial species such as Pseudomonas, Bacillus and Streptomyces spp. [3]. CLPs consist of a fatty acid tail linked to a short-to-medium-sized oligopeptide (up to $\sim 20$ amino acids), which takes part in a macrocyclic ring structure. The ring is closed by means of an amide or - in the majority of cases - an ester bond. The latter is also referred to as a depsi bond, and such CLPs are thus also called cyclic lipodepsipeptides. Unlike typical AMPs, most CLPs do not carry positive charges, being either uncharged (no ionizable functional groups) or negatively charged. Another feature is that most CLPs - at least for the portion involved in the macrocycle - already adopt a well-defined conformation in solution.

CLP-producing bacteria are found in numerous environments such as plants, soil, organic matter, water and even mammals. The large diversity in bacterial genera and strains that are able to produce CLPs, suggests that these compounds fulfill important functions for their producers [4]. Variations in the fatty acid moiety or in the number, type and configuration of the amino acids create an equally impressive diversity in CLP structures. Consequently, there is no single 'consensus' CLP structure but rather a common molecular blueprint. For example, in Pseudomonas spp., CLPs such as the bananamides, viscosins, orfamides, amphisins, xantholysins, etc., all appear to be similar isoforms with different peptide lengths, macrocycle sizes, and with some diversity in sequence and stereochemistry. There appears to be a degree of adaptability of the producing organisms in terms of CLP structure relative to environment, growth circumstances, etc.

\section{CLP research}

Reported antimicrobial activities for CLPs typically include antibacterial, antifungal and antiviral effects. Over the past few years, this has diversified to include insecticidal and anticarcinogenic activities. Apparently, the CLP blueprint can produce multifaceted biological agents, and much fundamental insight remains to be gained in the correlation between CLP sequence, structure and biological activity. Unfortunately, both the selectivity in biological effects investigated and the diversity in assay procedures prohibit any such analysis to be made (GeUdENS N ET 
aL., Unpublished Data). In our opinion, this seriously impedes the further development of CLPs as economically relevant agents in biomedical research. Moreover, we expect the situation to grow worse considering that the rate of discovery of novel metabolites is accelerating through the introduction of high-throughput approaches, such as mass-spectrometry-based molecular network indexing. The latter already allowed the discovery of the bananamides as a novel class of CLPs, and more are expected to emerge as these high-throughput techniques become mainstream [5]. The bottleneck is thus moving toward the biological evaluation.

Like for other metabolite classes, the scientific community has already invested in the development of dedicated databases for CLPs. For instance, the NORINE database catalogs CLP primary structures and their producing organisms [6]. However, depositing sequence, chemical and structural data are insufficient - it must be annotated with biological effects that are preferably extracted from a well-defined set of biological assays if we are to unlock the potential of CLPs. This will require the definition of a common set of microorganisms, reference strains (e.g., ATCC, NCPPB, BCCM, etc.) and pathogens together with established assaying procedures to characterize isolated CLPs. The latter could actively involve free open screening programs for antimicrobial activity such as performed by the Community for Antimicrobial Drug Discovery [7]. In time, this should lead to a database that can be mined for structure-function and structure-activity relationships, while the availability of uniform testing protocols would allow to immediately relate the functional repertoire of a novel, previously undescribed compound to the existing library.

\section{Investigating CLP mode of action}

A central research theme for AMPs - and CLPs are no exception - is elucidating the exact mechanism of their antimicrobial activity. For CLPs, the cellular membrane is clearly involved, although the exact mechanism remains unclear. Multiple modes of action have been described including pore formation, protein clustering, fluidity change, etc. Additionally, there may be not a single mechanism and multiple properties may be relevant. For instance, the CLP daptomycin is a so-called calcium-dependent antibiotic, containing a proposed calcium-binding Asp-X-Asp-Gly motif. In complex with $\mathrm{Ca}^{2+}$, daptomycin forms pore-like oligomers on the bacterial cytoplasmic membrane, leading to leakage of intracellular ions, followed by rapid cell death [8]. Moreover, daptomycin interacts with the negatively charged phosphatidylglycerol. This results in a membrane distortion and a delocalization of essential membrane proteins involved in cell division [9]. Membrane interaction studies often suffer from the use of homogenous model membranes, a far cry from the complex architecture of biological membranes. The recent advances in imaging methods which recently showed that daptomycin perturbs microdomains in bacterial cell membranes with concomitant metabolic interferences, bode well for the further understanding of the mechanism of action of AMPs in general and CLPs in particular in biologically relevant models. Indeed, the working mechanism of other cyclic lipodepsipeptides, involving the cellular membrane as primary target and which do not depend on the presence of $\mathrm{Ca}^{2+}$, is still poorly understood [10].

Oligomerization at the membrane interface appears to be a wide-spread phenomenon that potentially plays a relevant role in working mechanism of CLPs [11-13]. For CLPs of the viscosin group produced by Psendomonas spp., a structural proposal for the organization of a cylindrical self-associated complex was put forward whereby the monomer amphipathicity, which is conserved among the viscosin group, is thought to support the selfassembly [13-15]. In contrast, daptomycin appears to undergo a more 'disordered' self-assembly in the form of micelles [12]. Further structural studies concerning the nature of the self-assembled structures will be required to gain understanding in the biological relevance of this phenomenon. The biophysical tools are now being developed to distinguish/investigate these different mechanisms [16,17]. At the same time, recent developments allow efficient and rapid synthesis of CLPs, opening the door to synthetic variants that can be used for detailed structure-activity relationship studies [18,19]. Moreover, peptide synthesis can be used to introduce chemical modifications that improve upon biomedical properties, such as the therapeutic window, reduced haemolyticity, etc. [20,21]. Combined, these various developments will in the coming years lead to a breakthrough in uncovering the relation between the various CLP structural features, the antimicrobial activity and selectivity, and the working mechanism.

\section{Concerted action}

In the last decade, there has been a significant increase in our understanding of CLPs and their source bacteria. However, challenges will need to be overcome to ensure that the steady stream of scientific results concerning CLPs is accessible for investigation and is sufficiently correlated such that biomedical applications will manifest over time. This will require work in different domains, such as the screening of the biological diversity on different 
levels, including structural, microbiological, genetic and ecological and the study of their biophysical and chemical properties. A lot of progress has been made in recent years, and it is expected that this will continue at an increased rate. The CLP community, which so far has blended as isolated researcher groups in the larger world of AMP research, should seek each other out and come together to realize this exciting potential.

\section{Financial \& competing interests disclosure}

D Sinnaeve thanks the Research Foundation Flanders (FWO-Vlaanderen) for a postdoctoral fellowship grant. The authors have no other relevant affiliations or financial involvement with any organization or entity with a financial interest in or financial conflict with the subject matter or materials discussed in the manuscript apart from those disclosed.

No writing assistance was utilized in the production of this manuscript.

\section{References}

1. Bahar AA, Ren D. Antimicrobial peptides. Pharmaceuticals 6(12), 1543-1575 (2013).

2. Zasloff M. Antimicrobial peptides of multicellular organisms. Nature 415(6870), 389-395 (2002).

3. Sussmuth RD, Mainz A. Nonribosomal peptide synthesis-principles and prospects. Angew. Chem. Int. Edit. 56(14), 3770-3821 (2017).

4. Raaijmakers JM, De Bruijn I, Nybroe O, Ongena M. Natural functions of lipopeptides from Bacillus and Pseudomonas: more than surfactants and antibiotics. FEMS Microbiol. Rev. 34(6), 1037-1062 (2010).

5. Nguyen DD, Melnik AV, Koyama N et al. Indexing the Pseudomonas specialized metabolome enabled the discovery of poaeamide B and the bananamides. Nat. Microbiol. 2, 16197 (2017).

6. Caboche S, Pupin M, Leclere V, Fontaine A, Jacques P, Kucherov G. NORINE: a database of nonribosomal peptides. Nuc. Acids Res. 36, D326-D331 (2008).

7. Blaskovich MAT, Zuegg J, Elliott AG, Cooper MA. Helping chemists discover new antibiotics. ACS Infect. Dis. 1(7), 285-287 (2015).

8. Taylor SD, Palmer M. The action mechanism of daptomycin. Bioorg. Med. Chem. 24(24), 6253-6268 (2016).

9. Kleijn LHJ, Martin NI. The cyclic lipopeptide antibiotics. In: Topics in Medicinal Chemistry. Springer, Berlin, Germany (2017).

10. Geudens N, Nasir MN, Crowet JM et al. Membrane interactions of natural cyclic lipodepsipeptides of the viscosin group. Biochim. Biophys. Acta Biomembr. 1859(3), 331-339 (2017).

11. Hamley IW. Lipopeptides: from self-assembly to bioactivity. Chem. Commun. 51(41), 8574-8583 (2015).

12. Muraih JK, Pearson A, Silverman J, Palmer M. Oligomerization of daptomycin on membranes. Biochim. Biophys. Acta Biomembr. 1808(4), 1154-1160 (2011).

13. Sinnaeve D, Hendrickx PMS, Van Hemel J, Peys E, Kieffer B, Martins JC. The solution structure and self-association properties of the cyclic lipodepsipeptide pseudodesmin a support its pore-forming potential. Chem. Eur. J. 15(46), 12653-12662 (2009).

14. Geudens N, De Vleeschouwer M, Fehér K et al. Impact of a stereocentre inversion in cyclic lipodepsipeptides from the viscosin group: a comparative study of the viscosinamide and pseudodesmin conformation and self-assembly. ChemBioChem 15(18), 2736-2746 (2014).

15. Sinnaeve D, Delsuc MA, Martins JC, Kieffer B. Insight into peptide self-assembly from anisotropic rotational diffusion derived from ${ }^{13} \mathrm{C}$ NMR relaxation. Chem. Sci. 3(4), 1284-1292 (2012).

16. Lee TH, Hall KN, Aguilar MI. Antimicrobial peptide structure and mechanism of action: a focus on the role of membrane structure. Curr. Top. Med. Chem. 16(1), 25-39 (2016).

17. Müller A, Wenzel M, Strahl H et al. Daptomycin inhibits cell envelope synthesis by interfering with fluid membrane microdomains. Proc. Natl Acad. Sci. USA 113(45), e7077-e7086 (2016).

18. De Vleeschouwer M, Sinnaeve D, Van Den Begin J, Coenye T, Martins JC, Madder A. Rapid total synthesis of cyclic lipodepsipeptides as a premise to investigate their self-assembly and biological activity. Chem. Eur. J. 20(25), 7766-7775 (2014).

19. Lam HY, Zhang YF, Liu H et al. Total synthesis of daptomycin by cyclization via a chemoselective serine ligation. J. Am. Chem. Soc. 135(16), 6272-6279 (2013).

20. Dartois V, Sanchez-Quesada J, Cabezas E et al. Systemic antibacterial activity of novel synthetic cyclic peptides. Antimicrob. Agents Chemother. 49(8), 3302-3310 (2005).

21. Rabanal F, Grau-Campistany A, Vila-Farres X et al. A bioinspired peptide scaffold with high antibiotic activity and low in vivo toxicity. Sci. Rep. 5, 10558 (2015). 
